\section{Levofloxacino y rotura bilateral del tendón de Aquiles con evolución fatal}

\section{Sr. Director:}

Las nuevas quinolonas muestran unos perfiles de eficacia y comodidad en el tratamiento de las infecciones respiratorias adquiridas en la comunidad que han hecho que su uso se haya generalizado en la práctica habitual. No obstante, como ya destacan Lado y cols. (1), se debe valorar el aumento del riesgo de efectos adversos tendinosos descrito en algunos pacientes. Queremos aportar nuestra reciente experiencia en este aspecto.

Un hombre de 65 años, con antecedentes de enfermedad pulmonar obstructiva crónica severa, oxigenoterapia domiciliaria y tandas frecuentes de corticoides orales, consultó por caída con traumatismo torácico, tras la aparición de dolor intenso e impotencia funcional en ambos tendones aquíleos. Unos días antes se había pautado reposo, antiinflamatorios y suspensión de levofloxacino oral, por sospecha de tendinitis aquílea bilateral

La exploración física revelaba dolor costal izquierdo, crepitación subcutánea, solución de continuidad y tumefacción de ambos aquíleos (con hematoma aquíleo izdo) e imposibilidad para la flexión plantar de ambos pies (signo de Thompson). En la radiografía de tórax se apreciaban fractura de $2^{\circ}$ a $4^{\circ}$ arcos costales izdos, enfisema subcutáneo y un pequeño neumotórax izquierdo, mientras que la ecografía confirmaba la rotura aquílea bilateral. Se colocaron férulas de inmovilización (debido al elevado riesgo quirúrgico) y con tratamiento conservador se produjo reexpansión pulmonar total. Tras unos días de estabilidad clínica, el paciente presentó un progresivo deterioro respiratorio, sin recidiva del neumotórax y, pese al tratamiento de soporte, falleció.

Levofloxacino ha demostrado unos perfiles de eficacia, seguridad y comodidad que han hecho que su empleo haya aumentado en atención primaria y en el medio hospitalario. De hecho ha sido incluído como tratamiento de primera línea en los consensos de manejo de la neumonía adquirida en la comunidad (2) y de las reagudicaciones de los pacientes con EPOC (3). La tendinopatía por levofloxacino es infrecuente, inferior al $0,1 \%$ (4), y el de rotura tendinosa inferior a 4 por millón de prescripciones (5).
Pese a lo infrecuente de este efecto adverso, se deben tener en cuenta varios aspectos: a) medidas preventivas: evitar la prescripción indiscriminada y reconocer los factores de riesgo para la tendinopatía (corticoterapia, insuficiencia renal, edad avanzada, enfermedad reumática) (6); b) monitorizar los posibles efectos secundarios tras la prescripción, manteniendo un elevado índice de sospecha en el caso de aparición de síntomas osteomusculares; c) valorar el uso de técnicas complementarias de imagen (ecografía y/o RNM) ante la sospecha de afectación tendinosa; y d) si ésta se confirma además de retirar la fluoroquinolona e indicar reposo, probablemente sería aconsejable inmovilizar la articulación afectada.

En resumen, se debe valorar con cautela la prescripción y la monitorización de efectos secundarios tendinosos de las fluoroquinolonas en pacientes con factores de riesgo (especialmente ancianos y enfermos EPOC con corticoterapia).

\section{A. Sánchez Muñoz, F. J. Sanjuán Portugal, J. Naya Man-} chado, J. Castiella Herrero

Servicio de Medicina Interna. Fundación Hospital Calahorra. Calahorra. La Rioja

1. Lado F, Rodríguez C, Velasco M, Durán C, Moar B. Rotura parcial bilateral aquílea asociada a levofloxacino. An Med Interna (Madrid) 2005; 22: 28-30.

2. Grupo de Estudio de la Neumonía Adquirida en la Comunidad. Normativas para el diagnóstico y el tratamiento de la neumonía adquirida en la comunidad. Sociedad Española de Neumología y Cirugía Torácica (SEPAR). Arch Bronconeumol 2005; 41: 272-89.

3. Álvarez $\mathrm{F}$ et al. Uso de antimicrobianos en la exarcebación de la enfermedad pulmonar obstructiva crónica. Arch Bronconeumol 2002; 38: 81-9.

4. Carbon C. Levofloxacin adverse effects, data from clinical trials and pharmacovigilance. Therapie 2001; 56: 35-40.

5. Kahn JB. Latest industry information on the safety profile of levofloxacin in the US. Chemotherapy 2001; 47 (Supl. 3): 32-7.

6. Nuño FJ, Noval J, Suárez M, Guinea O. Dolor aquíleo e impotencia funcional en paciente con enfermedad pulmonar obstructiva crónica con neumonía. Rev Clin Esp 2001; 201: 539-540. 